BMJ Open

Sport \&

Exercise

Medicine

\title{
Effectiveness of a single platelet-rich plasma injection to promote recovery in rugby players with ankle syndesmosis injury
}

\author{
David J Samra, ${ }^{1}$ Amy D Sman, ${ }^{2,3}$ Katherine Rae, ${ }^{1}$ James Linklater, ${ }^{4}$ \\ Kathryn M Refshauge, ${ }^{2}$ Claire $\mathrm{E}$ Hiller $^{2}$
}

To cite: Samra DJ, Sman AD, Rae $\mathrm{K}$, et al. Effectiveness of a single platelet-rich plasma injection to promote recovery in rugby players with ankle syndesmosis injury. BMJ Open Sport Exerc Med 2015:0:e000033. doi:10.1136/bmjsem-2015000033

- Prepublication history for this paper is available online. To view these files please visit the journal online (http://dx.doi.org/10.1136/ bmjsem-2015-000033).

Accepted 18 August 2015

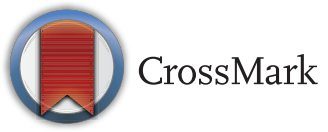

${ }^{1}$ The Sports Clinic, University of Sydney, Sydney, New South Wales, Australia ${ }^{2}$ Faculty of Health Sciences, University of Sydney, Sydney, New South Wales, Australia ${ }^{3}$ The Children's Hospital at Westmead, Sydney, New South Wales, Australia ${ }^{4}$ Castlereagh Imaging, Sydney, New South Wales, Australia

Correspondence to Dr David J Samra; davidjsamra@gmail.com

\section{ABSTRACT}

Aims: To determine whether a single ultrasoundguided platelet-rich plasma (PRP) injection into the anterior inferior tibiofibular ligament (AITFL) reduces the time for rugby athletes to return to function and match play following MRI confirmed ankle syndesmosis injury.

Methods: Cohort controlled pilot study. 10 Rugby Union players were recruited during the 2014 season, and consented to receive a single autologous PRP injection into the AITFL within 14 days of MRI confirmed ankle syndesmosis injury. A historical control group included 11 comparable Rugby Union players between 2011 and 2013 who were treated conservatively with the same inclusion criteria and rehabilitation protocol as the intervention group. Participants followed a standardised rehabilitation protocol involving simple milestones for progression. Early functional tests were performed 2 weeks after the removal of the CAM (controlled ankle motion) boot. Time to return to play was recorded. Repeat functional testing occurred within 1 week of return to play.

Results: Groups were comparable in anthropometrics, playing position and MRI injury severity. Time to return to play was significantly less in the intervention group $(p=0.048)$. Following return to play, athletes in the intervention group showed higher agility $(\mathrm{p}=0.002)$ and vertical jump $(p=0.001)$. There was a lower level of fear avoidance associated with rugby in the intervention group ( $\mathrm{p}=0.014$ )

Conclusions: This pilot study shows that, following ankle syndesmosis injury, a single autologous PRP injection may accelerate safe and successful return to Rugby Union, with improved functional capacity and reduced fear avoidance. It demonstrates the feasibility of a randomised controlled trial to further assess this therapy.

Trial registration number: ANZCTRN12614 000055606

\section{BACKGROUND}

Ankle syndesmosis injury (ASI) appears to be an increasingly common injury among

\section{What are the new findings?}

- This is the first study to report the effectiveness of a single PRP injection in ankle syndesmosis injuries for Rugby Union players, who appear to have a high incidence of this injury.

- PRP injections may accelerate safe return to play for Rugby Union players with non-surgical ankle syndesmosis injuries, when combined with appropriate rehabilitation.

- While a follow-on randomised controlled trial would be feasible and could provide firmer evidence of effectiveness, PRP obtained from simple systems and injected with ultrasound guidance appears to be safe and effective.

the sporting population. Several studies ${ }^{1-3}$ have suggested that these injuries have a higher incidence than once thought, and are probably underdiagnosed. Rugby Union seems to have a disproportionately high incidence of ASI, even compared with Rugby League $(0.89$ vs 0.46 injuries per $1000 \mathrm{~h}){ }^{4}$ While data are scarce in this field, the England Rugby Union Injury Surveillance Project ${ }^{3}$ reports a large increase in the incidence of ASI, ranking it third in overall incidence and first in days lost due to injury in 2013, with 145 days per 1000 match hours lost to injury (compared with 58 days in the 2012 season).

ASIs are considered more serious and complex, and result in longer delays to match play than lateral ligament injuries. ${ }^{2}$ While there is no consensus on optimal treatment, ${ }^{5}$ traditional treatment options have been limited either to conservative therapy with immobilisation, or to surgical fixation techniques. ${ }^{6}$ Time to return to play (RTP) after injury is often protracted; usually more than 6 weeks in rugby players we have treated, and on average four times longer 
than for lateral ligament injuries. ${ }^{7}$ Where conservative treatment is appropriate, new techniques are being sought to accelerate recovery.

In the last decade, basic science studies have suggested that treatments using autologous platelet-rich plasma (PRP) ${ }^{8}{ }^{9}$ have the potential to accelerate recovery and healing for various musculoskeletal injuries. Despite this, a recent systematic review concluded there is insufficient evidence to support the use of PRP injections in musculoskeletal soft tissue injuries; while pooled data did not demonstrate any harm from adverse events. ${ }^{10} \mathrm{~A}$ recent randomised controlled trial (RCT) assessed the influence of an ultrasound-guided PRP injection at diagnosis plus a second injection 1 week later, on RTP for athletes competing in a variety of different sports. ${ }^{11}$ Compared with athletes who received no injection, they demonstrated a shorter RTP, low residual pain on return and restoration of syndesmosis stability on ultrasound. An effective, minimally invasive treatment would be valuable to competitive and professional athletes with stable ASIs, who must otherwise weigh the risks and benefits of prolonged conservative recovery or surgery. Surgical intervention (with either screw or suture-button fixation) involves potential complications from the procedure (anaesthesia, bleeding, infection) and implant (failure or further surgery for removal), and an average of 6 weeks of postsurgical rest. $^{6}{ }^{12}$ The current pilot study was designed to obtain preliminary data about the effectiveness of PRP injection into the anterior inferior tibiofibular ligament (AITFL) in potentially accelerating safe RTP in a Rugby Union cohort, to inform the feasibility of conducting a fully powered RCT.

\section{METHODS}

We conducted a historical cohort-controlled study to assess the effectiveness of a single PRP injection for MRI-confirmed ASIs. Football clubs from the Sydney Rugby Union Premiership Competition were invited to participate. Ethics approval was obtained from the Human Research Ethics Committee at the University of Sydney, Australia (ID: 2014/439). Written informed consent was obtained from all participants.

Athletes referred by clubs for medical review were assessed by KR or DJS for ASI using clinical criteria described and evaluated in a previous diagnostic accuracy study. ${ }^{13}$ Key clinical features raising suspicion for syndesmosis injury included: tender syndesmotic ligaments on palpation, inability to single leg hop, positive dorsiflexion/external rotation stress test, positive squeeze test, pain out of proportion to the injury and sonographic evidence of injury. The absence of any of these features indicated a low probability of ASI. When clinical suspicion for ASI existed, MRI was performed within 14 days of injury and interpreted by a single radiologist (JL). MRI has excellent sensitivity and specificity for $\mathrm{ASI}^{14}$ and is far less invasive than arthroscopy, which is considered the reference standard. ${ }^{15}$ We used a classification system for ASI described by Sikka et al, ${ }^{16}$ which is applicable to our conservatively managed cohort. The grade is based on those ligamentous structures involved, as seen on MRI, that are thought to be injured sequentially with increasing tibiofibular displacement caused by increasing degrees of talar external rotation. ${ }^{17}$ Grade 1 describes isolated AITFL injury, grade 2 involves the addition of inferior interosseous ligament injury, grade 3 is the addition of posterior inferior tibiofibular ligament (PITFL) injury and grade 4 describes the addition of deltoid ligament injury. Static syndesmotic width $1 \mathrm{~cm}$ above the ankle joint anteriorly was also recorded in all cases, as syndesmotic width has been shown to have a significant correlation with recovery time. ${ }^{18}$ Exclusion criteria included: a low probability of ASI, previous ASI, previous ipsilateral fracture or surgery, concurrent injury likely to cause more prolonged disability, ankle fracture, frank tibiofibular diastasis on plain radiographs, osteochondral defect requiring surgery, concurrent acute grade 3 anterior talofibular ligament injury and greater than $4 \mathrm{~mm}$ tibiofibular separation on MRI.

In the 2014 season of the Sydney Rugby Union Premiership, 10 participants with MRI confirmed ASIs were recruited to the intervention group. Twelve patients were investigated using MRI, with 11 meeting diagnostic criteria for inclusion. One patient declined PRP injection, and one patient had lateral ligament rather than syndesmosis injury, as seen on MRI.

Participants in the intervention group underwent PRP injection within 14 days of injury. On enrolment, participants were asked to use paracetamol for analgaesia, and to refrain from taking non-steroidal anti-inflammatory drugs (NSAIDs) within 3 days of administration of PRP. This analgaesic restriction was intended to maximise the theoretical action of the PRP via platelet function. ${ }^{19}$ Using simple venesection, 1-2 mL of PRP was obtained from participants and put into sterile citrate tubes that were placed in a standard centrifuge at room temperature for $8 \mathrm{~min}$ at $3000 \mathrm{rpm}$. The portion of plasma just above the buffy coat was aspirated using a 21-gauge needle to obtain $0.5 \mathrm{~mL}$ of PRP per citrate tube, and no activating techniques were used. This procedure was based on an established technique, ${ }^{8}$ with the omission of chemical activation with calcium chloride. This modified method was validated in-house by JL to produce an average increased concentration of platelets by 3.3 times and of leucocytes by 2.9 times peripheral blood concentration. According to our recent survey of Fellows of the Australasian College of Sports Physicians (Samra D and Orchard J, unpublished data, 2014), the most common method to produce PRP is a manual centrifuge method without chemical or ultraviolet PRP activation techniques, which is akin to our method.

In administering the PRP, ultrasound guidance was used. The tear of the AITFL was identified, and an injection pathway that would avoid the superficial peroneal nerve was determined. Under sterile conditions, a 
25-gauge needle was used to inject $1-2 \mathrm{~mL}$ of local anaesthetic (1\% lignocaine) into the skin and superficial tissues. An individualised dose of $1-2 \mathrm{~mL}$ of the PRP was then injected into the precise region of the AITFL defect, so as to be contained and to not hydrodissect tissue further. JL performed all injections.

In addition to the PRP injection, the standard guidelines for rehabilitation remained consistent with milestones agreed on by medical staff (figure 1). While there are no evidence-based guidelines regarding the optimal conservative management of $\mathrm{ASI},{ }^{20}$ this approach is based on protection of injured tissue with relevant functional milestones to guide progression. All suspected acute ASIs were treated with rest, ice, compression, elevation, CAM (controlled ankle motion) boot immobilisation and modified pain-free weight-bearing. Regular weekly follow-up was observed, and participants were allowed to weight bear without a CAM boot once a painfree bilateral lunge (maximally bending at the knees while keeping both heels on the ground) and pain-free anterior ankle syndesmosis palpation were achieved. Running was only permitted once a pain-free single leg hop was achieved. In accordance with usual practice, all treating physiotherapists conducted a graded in-house sport-specific fitness test. This was considered the final milestone for RTP.

We compared the findings for the experimental study with a historical cohort recruited for a previous study. ${ }^{4}$ This cohort was obtained from injured Rugby Union players in the 2011-2013 seasons of the Sydney Rugby Union Premiership, who were recruited with the same criteria and treated by the same physician (DJS or KR).

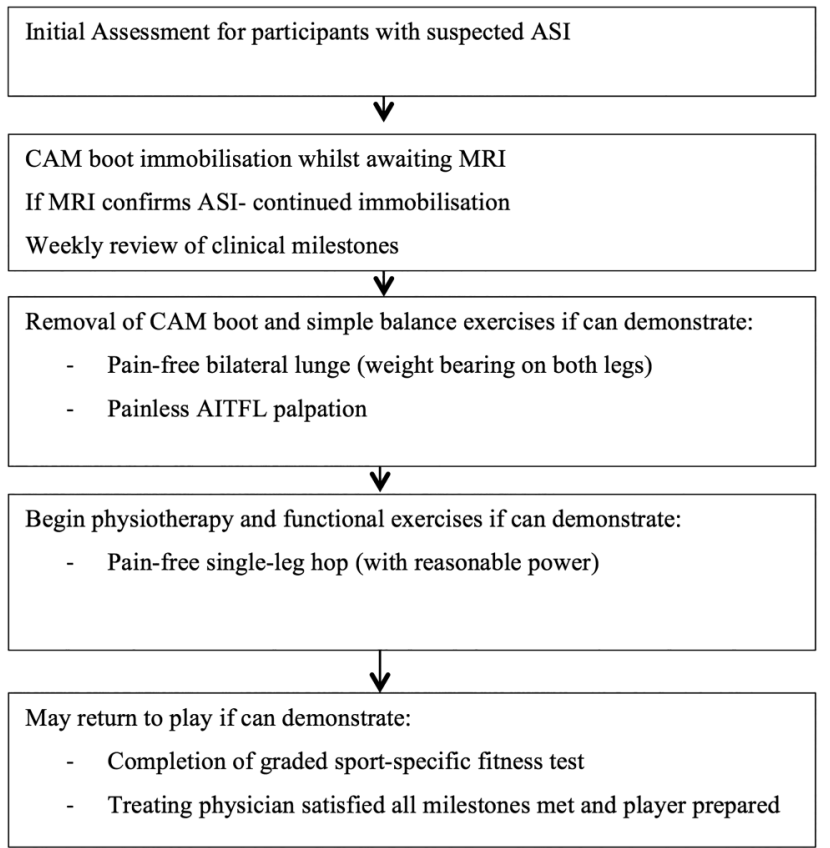

Figure 1 Rehabilitation protocol for ASI (AITFL, anterior inferior tibiofibular ligament; ASI, ankle syndesmosis injury; CAM, controlled ankle motion).
The intervention and historical cohorts were treated by three different physiotherapists at two clinics, each utilising the same sport-specific fitness test prior to RTP.

\section{Primary outcome}

The primary outcome measure was time to RTP. This was determined by measuring the number of days from the date of injury to the date of return to match play in competitive rugby within the Sydney Rugby Union Premiership.

\section{Secondary outcomes}

In the previous prospective cohort trial, the authors measured functional outcomes using the same protocol described in the present study. ${ }^{4}$ This testing protocol was adhered to for the intervention group (figure 2). Two weeks after participants were permitted to weight bear without the CAM boot, functional testing was conducted at the Early Testing Occasion. Outcomes measured included pain scores and functionally relevant measures of ankle range of motion, balance, power and fear of injury.

Pain was measured using a visual analogue scale (VAS) of $0-100 \mathrm{~mm}$. Scores prior to testing, after testing and following each individual test were recorded. This ensured test procedures did not cause undue discomfort, and provided a measure of symptomatic recovery.

Dorsiflexion range of motion was assessed using the weight-bearing lunge (WBL), a valid and reliable test of dorsiflexion range of motion. ${ }^{21}$ This was measured on both ankles, and normalised to foot length.

Balance was tested using the Star Excursion Balance Test (SEBT), a validated reliable measure. ${ }^{22}$ A composite score of the mean of the best attempt in each of three directions on each leg, normalised for leg length, was obtained.

Ankle power was assessed using a vertical jump (VJ), measured with a VJ mat. ${ }^{23}$

Fear of injury was assessed using a modified sport-specific Fear-Avoidance Beliefs Questionnaire ${ }^{24}$ (FABQ), in which higher scores are indicative of higher levels of fear; in order to assess confidence and readiness to RTP. There are three measures of fear-avoidance produced: physical activity-specific (FABQ pa), sport-specific (FABQ sport) and an overall score (FABQ total).

Within 1 week of return to match play, participants underwent further functional testing at the RTP Test Occasion. Tests included the aforementioned measures for the Early Test Occasion, with the addition of two further tests: the Illinois Agility Test and the Triple Hop test. These are considered valid measures of agility and ankle power, respectively. ${ }^{25} 26$

Analysis of data was performed using IBM SPSS Statistics V.22. Descriptive statistics were performed on all continuous variables including histogram frequencies, to assess normality. Baseline characteristics including age, weight, height and syndesmosis width on MRI 
Figure 2 Study flow chart (ASI, ankle syndesmosis injury; PRP, platelet-rich plasma).
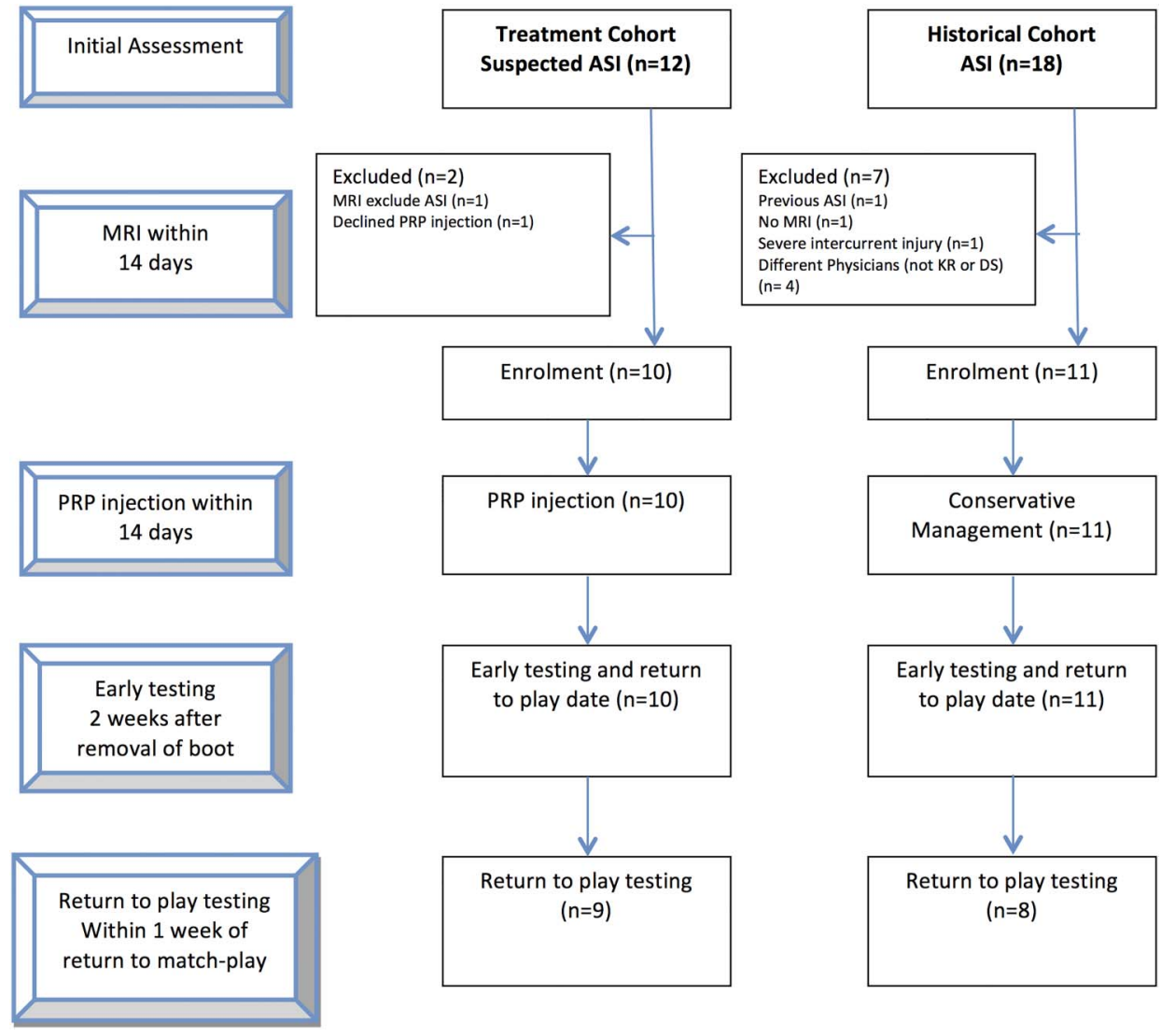

were analysed using Student $\mathrm{t}$ tests to detect any significant differences between cohorts. Similarly, quantitative outcome measures of pain, function and time to RTP were analysed to compare groups using Student t tests of independent samples. Where data were not normally distributed, non-parametric tests were used to detect differences between groups.

\section{RESULTS}

The intervention and historical groups were comparable in all baseline demographic data $(p>0.05$ for all characteristics; table 1), and there were similar numbers of Forwards and Backs in each group. Grade 3 injuries predominated in both groups, with a shift toward more grade 4 injuries and less grade 3 injuries in the intervention group. Overall, the grade of injury was similar, and groups were comparable in the severity of their injury as measured by static width on MRI ( $p=0.23)$.

\section{Primary outcome}

The mean difference in RTP was 20.7 days less for the intervention group compared with the historical control, $\mathrm{p}=0.048,95 \%$ CI (0.04 to 41.3 ).

\section{Secondary outcomes}

At the Early Test Occasion, athletes in the intervention group had higher VJ height $(\mathrm{p}=0.01)$ and lower pain scores during this activity compared with the historical group. There were no significant differences between groups in terms of pain scores during other tests, other functional tests or fear-avoidance beliefs (table 2).

At the RTP Test Occasion, athletes in the intervention group showed superior agility on the Illinois agility test by a mean of $3.4 \mathrm{~s}, \mathrm{p}=0.002,95 \%$ CI ( 1.6 to 5.1 ). VJ was a mean of $11 \mathrm{~cm}$ higher, $\mathrm{p}=0.001,95 \%$ CI (5.6 to 16.4 ). There was a reduced level of fear-avoidance beliefs associated with Rugby Union in the intervention group, $\mathrm{p}=0.014$ (table 2). Three participants from the historical group and one from the intervention group did not

\begin{tabular}{|c|c|c|c|}
\hline Variables & $\begin{array}{l}\text { Intervention } \\
(\mathrm{n}=10)\end{array}$ & $\begin{array}{l}\text { Historical } \\
(n=11)\end{array}$ & $\begin{array}{l}p \\
\text { Value }\end{array}$ \\
\hline Age (years) & $20.6(1.8)$ & $20.4(2.7)$ & 0.815 \\
\hline Height (cm) & 183.4 (6.9) & $181.9(4.5)$ & 0.569 \\
\hline Weight (kg) & $94.9(13.4)$ & 93.6 (11.9) & 0.823 \\
\hline $\begin{array}{l}\text { Syndesmosis width } \\
\text { on MRI }(\mathrm{mm})\end{array}$ & $2.1(0.6)$ & $2.5(0.9)$ & 0.220 \\
\hline Position: Forwards & 7 & 7 & \\
\hline Position: Backs & 3 & 4 & \\
\hline \multicolumn{4}{|l|}{ Injury grade $e^{16}$} \\
\hline Grade 1 & 0 & 0 & \\
\hline Grade 2 & 1 & 1 & \\
\hline Grade 3 & 7 & 10 & \\
\hline Grade 4 & 2 & 0 & \\
\hline
\end{tabular}


Table 2 Performance on functional tests at early testing and RTP testing (mean \pm SD)

\begin{tabular}{|c|c|c|c|}
\hline Primary outcome & Intervention $(n=10)$ & Historical $(n=11)$ & p Value \\
\hline Time to RTP (days) & $48.6(11.7)$ & $69.3(29.1)$ & $0.048^{*}$ \\
\hline Early testing variables & Intervention $(n=10)$ & Historical $(n=11)$ & p Value \\
\hline$\overline{V J}(\mathrm{~cm})$ & $58.2(8.5)$ & $45.9(9.8)$ & $0.014^{*}$ \\
\hline VJ pain (VAS) & $16.0(16.5)$ & $40.0(23.9)$ & $0.032^{*}$ \\
\hline WBL (cm) & $34.6(4.3)$ & $34.3(3.7)$ & 0.879 \\
\hline Pain pretesting (VAS) & $1.0(3.2)$ & $2.8(6.4)$ & 0.418 \\
\hline FABQ pa & $14.2(4.3)$ & $15.6(5.0)$ & 0.513 \\
\hline FABQ sport & $22.9(6.0)$ & $26.4(3.8)$ & 0.138 \\
\hline FABQ total & $52.0(13.1)$ & $57.1(10.7)$ & 0.353 \\
\hline RTP testing variables & Intervention $(n=9)$ & Historical $(\mathrm{n}=8)$ & p Value \\
\hline Illinois agility (s) & $15.0(1.2)$ & $18.4(2.1)$ & $0.002^{*}$ \\
\hline VJ (cm) & $52.9(5.7)$ & $63.9(4.6)$ & $0.001^{*}$ \\
\hline WBL (cm) & $36.8(3.3)$ & $36.2(3.4)$ & 0.731 \\
\hline SEBT $(\mathrm{cm})$ & $77.3(4.7)$ & $75.3(4.6)$ & 0.374 \\
\hline Triple hop (m) & $5.5(0.7)$ & $5.4(0.5)$ & 0.744 \\
\hline FABQ pa & $9.3(5.4)$ & $10.2(4.7)$ & 0.701 \\
\hline FABQ sport & $14.4(7.2)$ & $22.4(5.8)$ & $0.029^{*}$ \\
\hline FABQ total & $27.0(16.9)$ & $39.8(11.4)$ & 0.087 \\
\hline
\end{tabular}

${ }^{*}$ Significant $\mathrm{p} \leq 0.05$.

FABQ, Fear-Avoidance Beliefs Questionnaire (subscores: pa, physical activity-specific score; sport, sports-specific score; total, overall score); RTP, return to play; SEBT, Star Excursion Balance Test; VAS, visual analogue scale; VJ, vertical jump; WBL, weight-bearing lunge.

attend RTP testing. These participants did not differ significantly from their cohort in any baseline characteristics or injury severity. No loss to follow-up occurred for RTP date and recovery time data.

No participants in either group reported resting pain at the RTP Test Occasion, however, mild pain was provoked during testing in four athletes from the intervention group, compared with six from the historical control group. On routine medical follow-up of the intervention group 3 months after RTP, there were no reports of recurrence or ongoing symptoms. No participant underwent ankle surgery.

Since there were no complications directly attributable to the PRP treatment, this appears to be a safe therapy for ASIs. There were no reports of adverse events in the immediate period of follow-up performed at 2-3 days postinjection for participants in the intervention group. Later in their recovery, two athletes had unexpected adverse events. One participant was found to have a below-knee deep vein thrombosis diagnosed early in his management. This occurred 2 weeks after the PRP injection and thought to be related to immobilisation. Another participant had a non-contact ACL rupture of his ipsilateral knee on his first game returning to rugby. This player, as with all participants, underwent graded fitness testing in order to assess safety to RTP.

\section{DISCUSSION}

This is the first study to investigate the effectiveness of an ultrasound-guided PRP injection in accelerating RTP in Rugby Union players. We found that participants who received a single PRP injection took significantly less time to RTP than controls. This is in keeping with the findings of Laver et $a l,{ }^{11}$ who found that athletes from a variety of sports returned to play significantly faster and with lower levels of residual pain. In contrast to that study, the current study recruited a homogeneous single sport cohort. Furthermore, we tested a simple PRP protocol involving a manual single spin procedure and single injection (rather than Endoret), and we recorded functional outcome measures to confirm safe and appropriate return to sport. The findings of reduced recovery time following PRP injection are supported by significantly higher measures of ankle power and agility, indicative of a better capacity for performance on RTP.

At the Early Test Occasion, athletes in the intervention group following PRP injection had higher VJ scores than those in the historical control group. There were no significant differences in any other outcomes, including pain scores, balance or fear-avoidance beliefs. Considering that injury severity was comparable between groups, this may represent an early improvement in function associated with a more stable ankle mortise, since jumping places the ankle mortise under considerable stress. Functional superiority of the intervention group was maintained on the RTP Test Occasion. Scores on both the Illinois Agility and VJ tests were significantly higher in the intervention group. Maintenance of greater mortise stability may be responsible for this effect, as the PRP administered is thought to increase stability via augmented tissue healing. ${ }^{11}$ While some authors cite the trophic effects of growth factors in accelerating the proliferative phase of tissue healing, clinical researchers such as J Fitzpatrick (unpublished data, 2013) have suggested that a sclerosing effect may 
promote tissue fibrosis and stiffening due to the increased glucose concentration of PRP. In the future, a dynamic imaging test such as serial ultrasound may prove beneficial in quantifying the functional stability of the ankle mortise following such treatment. ${ }^{27}$

This study appears to demonstrate that a single ultrasound-guided PRP injection into the ASI is safe and effective in accelerating recovery. This is highly applicable to clinical practice for the management of ASIs in Rugby Union players. We applied a single PRP injection produced from a single centrifuge spin in order to maximise external validity. A single injection is easier for the patient and clinician. Furthermore, our recent survey (Samra D and Orchard J, unpublished data, 2014) highlighted that most Australasian Sports Physicians do not use commercial kits, or activation methods (such as ultraviolet light or calcium chloride) to produce PRP. Team physicians trained in ultrasound-guided injections, with minimal additional training, equipment or cost, could easily apply the simple method of PRP preparation we tested. Nonetheless, there remain wide differences in methods of preparing PRP, which could limit the generalisability of our results.

The use of an historical control is a limiting factor in this study, as it does not involve placebo control or randomisation. Since participants were not blinded to the treatment received, there is the possibility of a placebo effect in the intervention group. However, despite the lack of randomisation, group characteristics were highly comparable, with all participants drawn from a similar competitive level of rugby (table 1). All athletes were Sydney Rugby Union Premiership players and were included using the same criteria, same treatment progression milestones and the same treating physicians. Baseline characteristics, player position, grade of injury and syndesmosis width on MRI were also highly correlated. For the purposes of this pilot study, a historical control group was utilised to ensure realistic recruitment targets. This study indicates that an RCT with adequate power to compare PRP injection with placebo injection would be feasible.

\section{Conclusions}

A single autologous PRP injection appears to be a safe intervention that may accelerate successful return to Rugby, with improved functional capacity and reduced fear avoidance following ASI. Although it may be practically difficult to recruit elite athletes into placebo control groups, further trials with randomisation and placebo control methodology are feasible and warranted.

Twitter Follow David Samra at @dave_samra

Acknowledgements The authors thank the staff and players at Sydney University Rugby Club, Eastern Suburbs Rugby Club and other clubs of the Sydney Rugby Union Premiership for their assistance and participation in the study. They thank Dr Jenny Peat for providing consultation with statistical expertise. They also thank Zheng Cao for her contributions to the refinement of the study manuscript.
Contributors DJS, KR and ADS conceived the study. DJS and ADS initiated the study design. DJS, KR and JL performed clinical treatments and review for implementation of the study protocol. ADS and DJS performed initial and return to play testing protocols. DJS conducted the primary statistical analysis. All the authors contributed to refinement of the study protocol and approved the final manuscript.

Funding Financial support was received to fund equipment for functional testing and procedural costs associated with platelet-rich plasma injections. This was provided through an AIS Performance Research Funding Grant. DJS is the AIS Performance Research Funding grant holder.

Competing interests None declared.

Ethics approval Sydney University Human Research Ethics Committee, Human ethics number 2013/439.

Provenance and peer review Not commissioned; externally peer reviewed.

Data sharing statement All the data generated from this study will be freely available to coauthors and future researchers involved in ankle syndesmosis research at the Arthritis and Musculoskeletal Research Group, University of Sydney.

Open Access This is an Open Access article distributed in accordance with the Creative Commons Attribution Non Commercial (CC BY-NC 4.0) license, which permits others to distribute, remix, adapt, build upon this work noncommercially, and license their derivative works on different terms, provided the original work is properly cited and the use is non-commercial. See: http:// creativecommons.org/licenses/by-nc/4.0/

\section{REFERENCES}

1. Bloemers $F$, Bakker $F$. Acute ankle syndesmosis injury in athletes. Eur J Trauma 2006;32:350-6.

2. Gerber JP, Williams GN, Scoville CR, et al. Persistent disability associated with ankle sprains: a prospective examination of an athletic population. Foot Ankle Int 1998;19:653-60.

3. England Professional Rugby Injury Surveillance Project. Secondary England Professional Rugby Injury Surveillance Project. http://www. rfu.com/takingpart/ /media/files/2014/takingpart/injury_audit_report_ 2014 .ashx

4. Sman AD, Hiller CE, Rae K, et al. Predictive factors for ankle syndesmosis injury in football players: a prospective study. J Sci Med Sport 2014;17:586-90.

5. Jones MH, Amendola A. Syndesmosis sprains of the ankle: a systematic review. Clin Orthop Relat $R$ 2007:455:173-5.

6. Taylor DC, Tenuta JJ, Uhorchak JM, et al. Aggressive surgical treatment and early return to sports in athletes with grade III syndesmosis sprains. Am J Sport Med 2007;35:1833-8.

7. Sman AD, Hiller CE, Rae K, et al. Prognosis of ankle syndesmosis injury. Med Sci Sports Exerc 2014;46:671-7.

8. Anitua E, Andia I, Ardanza B, et al. Autologous platelets as a source of proteins for healing and tissue regeneration. Thromb Haemost 2004:91:4-15.

9. Woodall J Jr, Tucci M, Mishra A, et al. Cellular effects of platelet rich plasmainterleukin1 release from PRP treated macrophages. Biomed Sci Instrum 2008;44:489-94.

10. Moraes VY, Lenza M, Tamaoki MJ, et al. Platelet-rich therapies for musculoskeletal soft tissue injuries. Cochrane Database Syst Rev 2014;4:CD010071.

11. Laver L, Carmont MR, McConkey MO, et al. Plasma rich in growth factors (PRGF) as a treatment for high ankle sprain in elite athletes: a randomized control trial. Knee Surg Sports Traumatol Arthrosc 2014. [Published Online First: 19/06/2014].

12. Porter DA, Jaggers RR, Barnes AF, et al. Optimal management of ankle syndesmosis injuries. Open Access J Sports Med 2014:5:173-82.

13. Sman AD, Hiller CE, Rae K, et al. Diagnostic accuracy of clinical tests for ankle syndesmosis injury. Br J Sports Med 2015;49:323-9.

14. Oae K, Takao M, Naito K, et al. Injury of the tibiofibular syndesmosis: value of MR imaging for diagnosis. Radiology 2003;227:155-61.

15. Han SH, Lee JW, Kim S, et al. Chronic tibiofibular syndesmosis injury: the diagnostic efficiency of magnetic resonance imaging and comparative analysis of operative treatment. Foot Ankle Int 2007:28:336-42.

16. Sikka RS, Fetzer GB, Sugarman E, et al. Correlating MRI findings with disability in syndesmotic sprains of NFL players. Foot Ankle Int 2012:33:371-8 
17. Zalavras C, Thordarson D. Ankle syndesmotic injury. J Am Acad Orthop Surg 2007;15:330-9.

18. Howard DR, Rubin DA, Hillen TJ, et al. Magnetic resonance imaging as a predictor of return to play following syndesmosis (high) ankle sprains in professional football players. Sports Health 2012;4:535-43.

19. Ikeda $Y$. The effect of ibuprofen on platelet function in vivo. Keio $J$ Med 1977;26:213-22.

20. Amendola A, Williams G, Foster D. Evidence-based approach to treatment of acute traumatic syndesmosis (high ankle) sprains. Sports Med Arthrosc 2006;14:232-6.

21. Konor MM, Morton S, Eckerson JM, et al. Reliability of three measures of ankle dorsiflexion range of motion. Int J Sports Phys Ther 2012;7:279-87.

22. Plisky PJ, Rauh MJ, Kaminski TW, et al. Star Excursion Balance Test as a predictor of lower extremity injury in high school basketball players. J Orthop Sports Phys Ther 2006;36:911-19.
23. Hutchison A, Stone AL. Validity of alternative field system for measuring vertical jump height. J Exerc Physiol 2009;12: 6-11.

24. Simon CB, Stryker SE, George SZ. Comparison of work-related fear-avoidance beliefs across different anatomical locations with musculoskeletal pain. J Pain Res 2011;4:253-62.

25. Hachana Y, Chaabene H, Nabli MA, et al. Test-retest reliability, criterion-related validity, and minimal detectable change of the Illinois agility test in male team sport athletes. J Strength Cond Res 2013;27:2752-9.

26. Hamilton RT, Shultz SJ, Schmitz RJ, et al. Triple-hop distance as a valid predictor of lower limb strength and power. J Athl Train 2008;43:144-51.

27. Mei-Dan O, Carmont M, Laver L, et al. Standardization of the functional syndesmosis widening by dynamic U.S examination. BMC Sports Sci Med Rehabil 2013;5:9. 